\title{
Comprehensive Analysis of HD 105, A Young Solar System Analog
}

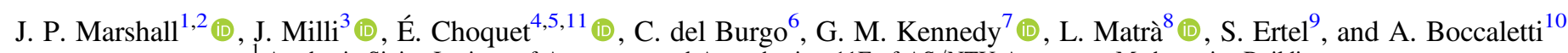 \\ Academia Sinica Institute of Astronomy and Astrophysics, 11F of AS/NTU Astronomy-Mathematics Building, \\ No.1, Section 4, Roosevelt Road, Taipei 10617, Taiwan, People's Republic of China \\ ${ }^{2}$ Computational Engineering and Science Research Centre, University of Southern Queensland, Toowoomba, QLD 4350, Australia \\ ${ }^{3}$ European Southern Observatory (ESO), Alonso de Córdova 3107, Vitacura, Casilla 19001, Santiago, Chile \\ ${ }^{4}$ Department of Astronomy, California Institute of Technology, 1200 E. California Boulevard, MC 249-17, Pasadena, CA 91125, USA \\ ${ }^{5}$ Jet Propulsion Laboratory, California Institute of Technology, 4800 Oak Grove Drive, Pasadena, CA 91109, USA \\ ${ }^{6}$ Instituto Nacional de Astrofísica, Óptica y Electrónica, Luis Enrique Erro 1, Sta. Ma. Tonantzintla, Puebla, Mexico \\ ${ }^{7}$ Department of Physics, University of Warwick, Coventry CV4 7AL, UK \\ ${ }_{9}^{8}$ Harvard-Smithsonian Center for Astrophysics, 60 Garden Street, Cambridge, MA 02138, USA \\ ${ }^{9}$ Steward Observatory, University of Arizona, 933 N Cherry Avenue, Tucson, AZ 85719, USA \\ ${ }^{10}$ LESIA, Observatoire de Paris, PSL Research University, CNRS, Sorbonne Universités, \\ UPMC Univ. Paris 06, Université Paris Diderot, Sorbonne Paris Cité, France \\ Received 2018 August 22; revised 2018 October 12; accepted 2018 October 25; published 2018 December 6
}

\begin{abstract}
HD 105 is a nearby, pre-main-sequence G0 star hosting a moderately bright debris disk $\left(L_{\text {dust }} / L_{\star} \sim 2.6 \times 10^{-4}\right.$ ). The star and its surroundings might therefore be considered an analog of the young solar system. We refine the stellar parameters based on an improved Gaia parallax distance and identify it as a pre-main-sequence star with an age of $50 \pm 16 \mathrm{Myr}$. The circumstellar disk was marginally resolved by Herschel/PACS imaging at far-infrared wavelengths. Here, we present an archival ALMA observation at $1.3 \mathrm{~mm}$, revealing the extent and orientation of the disk. We also present Hubble Space Telescope (HST)/NICMOS and VLT/SPHERE near-infrared images, where we recover the disk in scattered light at the $\geqslant 5 \sigma$ level. This was achieved by employing a novel annular averaging technique and is the first time this has been achieved for a disk in scattered light. Simultaneous modeling of the available photometry, disk architecture, and detection in scattered light allow better determination of the disk's architecture, and dust grain minimum size, composition, and albedo. We measure the dust albedo to lie between 0.19 and 0.06, the lower value being consistent with Edgeworth-Kuiper Belt objects.
\end{abstract}

Key words: circumstellar matter - planetary systems - stars: individual (HD 105)

\section{Introduction}

Through the results of the Kepler transit-based exoplanet survey, we are now aware that exoplanets are near-ubiquitous (Coughlin et al. 2016; Foreman-Mackey et al. 2016). Circumstellar debris, most commonly identified through excess emission at infrared wavelengths (Matthews et al. 2014), is less frequently found. However, this is more severely biased and limited by current instrumental sensitivity. A detection rate of $20 \%$ has been recorded for cool disks around nearby FGK-type stars (Eiroa et al. 2013; Montesinos et al. 2016), with a slightly higher detection rate around A-type stars (Thureau et al. 2014). A tentative trend for a higher incidence of debris disks is seen around stars with subsolar metallicities hosting low-mass planets (Wyatt et al. 2012; Marshall et al. 2014b). For highmass planets, a recent study of disk-host systems has identified a tentative correlation between the presence of $>5 M_{\text {Jup }}$ planets and debris disks (Meshkat et al. 2017). A study of exoplanet host stars and debris disks reveals a trend between these components of planetary systems being seen together (Matthews et al. 2014). However, no evidence of correlations between these properties has been seen in larger stellar samples, while potentially attributable to the paucity of information on both faint debris disks and low-mass planets around nearby stars, the presence of real correlations cannot be ruled out due to sample construction (Moro-Martín et al. 2015).

A critical component in developing an understanding of the diversity of architectures exhibited by known planetary systems

${ }^{11}$ Hubble Fellow. is to obtain multiwavelength resolved images of their dusty debris disks (e.g., Ertel et al. 2014; Marshall et al. 2014a, 2016; Hengst et al. 2017). Using a sample of 34 resolved debris disks, a relationship between stellar luminosity and dust properties has been identified, showing that the dust grains around higher luminosity stars are closer to emitting like blackbodies (Pawellek et al. 2014). Fitting that observed relationship with a range of dust material compositions further identified that a mixture of astronomical silicate and water ice provided the best fit to the distribution of spatially resolved disks (Pawellek \& Krivov 2015). The presence of icy material in cool debris disks has also been inferred through the modeling of continuum emission of several spatially resolved debris disks, supporting the adoption of icy materials in the analysis of debris dust (Lebreton et al. 2012; Morales et al. 2016). More recently, a relationship between stellar luminosity and disk radius has been measured for a similar-sized sample of debris disks imaged at submillimeter wavelengths (Matrà et al. 2018a). However, no evidence was found for the trend of disk radius relative to blackbody radius identified at far-infrared wavelengths. This may be attributed to the low spatial resolution and bias toward higher luminosity stars of the far-infrared sample, making it less representative overall.

Analysis of the infrared continuum emission from debris dust can only take our understanding so far. To obtain insight into the dust grain composition and structure (porosity), we require measurement of the silicate features at mid-infrared wavelengths (e.g., Beichman et al. 2006; Chen et al. 2006; Lawler et al. 2009; Johnson et al. 2012; Mittal et al. 2015) and/ or measurement of scattered light from the disk (either total 
intensity or polarized light). A review of recent advancements in the interpretation of scattered light imaging of debris disks is presented in Hughes et al. (2018). In the case of silicate features, a minority of debris disk-host stars exhibit features in their mid-infrared spectra (Beichman et al. 2006; Chen et al. 2014), suggesting that the constituent dust grains are large $(>10 \mu \mathrm{m})$ and/or cold. In the latter case, the scattered light brightness is poorly correlated with expectations from the disk brightness in continuum emission (e.g., Schneider et al. 2014) but for those disks that have been imaged in scattered light, determination of the dust optical and scattering properties has been possible (e.g., Graham et al. 2007; Krist et al. 2010; Rodigas et al. 2012, 2014; Soummer et al. 2014; Choquet et al. 2016; Schneider et al. 2016). Complementary to the properties of solid material in debris disks, the recent detections of a gaseous component to some of these systems (Greaves et al. 2016) provide insight into the volatile content of the dust parent bodies (e.g., Matrà et al. 2017) and its origins (Kral et al. 2017).

For Sun-like stars, from which we may draw parallels with the evolution of the solar system, there exist only a few cases with comprehensive multiwavelength data sets to support such detailed analyses, e.g., HD 15115 (Debes et al. 2008; MacGregor et al. 2015), HD 61005 (Hines et al. 2007; Maness et al. 2009; Buenzli et al. 2010; Olofsson et al. 2016), HD 107146 (Ertel et al. 2011; Ricci et al. 2015; Marino et al. 2018), HD 207129 (Krist et al. 2010; Marshall et al. 2011; Löhne et al. 2012), HIP 17439 (Ertel et al. 2014; Schüppler et al. 2014), and HD 10647 (Liseau et al. 2010; Schüppler et al. 2016). The addition of more targets to this list, particularly within the narrow range of stellar spectral types representative of solar analogs (i.e., early G-type main-sequence stars cf. a broad range of F6 to $\mathrm{K} 0$ spectral types), is therefore important to understand the diversity of potential outcomes for planet formation processes. The relative novelty of the solar system may therefore be determined by comparison to the range of properties exhibited by analogous debris disk systems.

HD 105 is a young, Sun-like star at a distance of $40 \mathrm{pc}$ that hosts a moderately bright debris disk $\left(L_{\text {dust }} / L_{\star} \sim 2.6 \times 10^{-4}\right)$. HD 105 is a member of the Tucana-Horologium association and has a well-established age of $28 \pm 4$ Myr (Torres et al. 2000; Zuckerman \& Webb 2000). Its debris disk was reported as being marginally resolved in far-infrared Herschel/PACS imaging observations and found to have a radius of $\sim 50$ au (Donaldson et al. 2012). With a spectral type of G0, HD 105 might therefore be considered an analog of the young solar system.

Here we model the continuum emission and structure of HD 105's debris disk using a combination of far-infrared and submillimeter photometry obtained from a new reduction of archival Herschel/PACS and SPIRE images, and a spatially resolved archival image of the disk at millimeter wavelengths from ALMA. We complement this approach with analysis of near-infrared images from HST/NICMOS and VLT/SPHERE in order to constrain the dust optical properties through scattered light.

In Section 2, we present the imaging and photometric data compiled to model both the stellar and disk components of this system. In Section 3, we lay out our approach to analyzing the combined data set and state our findings. In Section 4 the results, as well as their impact on our understanding of this system, are put in context. Finally, in Section 5, we summarize our findings and detail the conclusions of this work.
Table 1

Photometry Used in Disk Modeling

\begin{tabular}{|c|c|c|c|}
\hline $\begin{array}{l}\text { Wavelength } \\
(\mu \mathrm{m})\end{array}$ & $\begin{array}{l}\text { Flux } \\
(\mathrm{mJy})\end{array}$ & $\begin{array}{l}\text { Instrument/ } \\
\text { Filter }\end{array}$ & References \\
\hline 0.349 & $891 \pm 2$ & Strömgren $u$ & 1 \\
\hline 0.411 & $2071 \pm 6$ & Strömgren $b$ & 1 \\
\hline 0.440 & $1897 \pm 14$ & Johnson $B$ & 2 \\
\hline 0.467 & $2989 \pm 6$ & Strömgren $v$ & 1 \\
\hline 0.546 & $3669 \pm 2$ & Strömgren $y$ & 1 \\
\hline 0.550 & $3499 \pm 26$ & Johnson $V$ & 2 \\
\hline 0.790 & $4468 \pm 41$ & Cousins $I$ & 3 \\
\hline 1.235 & $4139 \pm 73$ & 2MASS $J$ & 4 \\
\hline 1.662 & $3425 \pm 73$ & 2MASS $H$ & 4 \\
\hline 2.159 & $2383 \pm 42$ & 2MASS $K_{s}$ & 4 \\
\hline 3.40 & $1152 \pm 122$ & WISE W1 & 5 \\
\hline 4.60 & $699 \pm 24$ & WISE W2 & 5 \\
\hline 9.00 & $189 \pm 13$ & AKARI/IRC9 & 6 \\
\hline 12.0 & $114 \pm 6$ & WISE W3 & 5 \\
\hline 22.0 & $35 \pm 2$ & WISE W4 & 5 \\
\hline 24.0 & $29 \pm 1$ & Spitzer/MIPS & 7 \\
\hline 31.0 & $22 \pm 7$ & Spitzer/IRS & 8 \\
\hline 60.0 & $143 \pm 3$ & $I S O / \mathrm{PHT}$ & 9 \\
\hline 70.0 & $141 \pm 10$ & Spitzer/MIPS & 7 \\
\hline 70.0 & $132 \pm 8$ & Herschel/PACS & 10 \\
\hline 90.0 & $167 \pm 8$ & $I S O / \mathrm{PHT}$ & 9 \\
\hline 100.0 & $168 \pm 8$ & Herschel/PACS & 10 \\
\hline 160.0 & $112 \pm 9$ & Herschel/PACS & 10 \\
\hline 250.0 & $57 \pm 10$ & Herschel/SPIRE & 11 \\
\hline 350.0 & $38 \pm 15$ & Herschel/SPIRE & 11 \\
\hline 880.0 & $10.7 \pm 5.9$ & APEX/LABOCA & 12 \\
\hline 1300.0 & $2.0 \pm 0.4$ & ALMA & 11 \\
\hline 9000.0 & $0.042 \pm 0.014$ & ATCA & 13 \\
\hline
\end{tabular}

References. 1. Paunzen (2015); 2. Mermilliod et al. (1997); 3. Perryman et al. (1997); 4. Cutri et al. (2003); 5. Wright et al. (2010); 6. Ishihara et al. (2010); 7. Carpenter et al. (2009); 8. Chen et al. (2014); 9. Spangler et al. (2001); 10. Donaldson et al. (2012); 11. This work; 12. Nilsson et al. (2010); 13. Marshall et al. (2017).

\section{Observations}

We have compiled broadband photometric data from optical to millimeter wavelengths to construct the spectral energy distribution (SED) of HD 105. These previous observations, combined with the ALMA, VLT/SPHERE, and HST/NICMOS imaging data presented here, facilitate comprehensive modeling of the HD 105 system. A summary of the photometry used in the modeling process is presented in Table 1.

At optical wavelengths, we use the Strömgren data from Paunzen (2015), Johnson $B V$ and Cousins $I$ data from Mermilliod et al. (1997), near-infrared 2MASS $J H K_{s}$ (Cutri et al. 2003), and WISE W1 and W2 fluxes (Wright et al. 2010) to scale the model stellar photosphere and gauge its contribution to the total emission.

In the mid-infrared, the WISE W3 $(12 \mu \mathrm{m})$ and W4 $(22 \mu \mathrm{m})$ fluxes are complemented by $A K A R I / I R C 9 \mu \mathrm{m}$ data point (Ishihara et al. 2010), a Spitzer/MIPS measurement at $24 \mu \mathrm{m}$, and Spitzer/IRS photometry at 13 and $31 \mu \mathrm{m}$. The Spitzer/IRS spectrum was obtained from CASSIS ${ }^{12}$ (Lebouteiller et al. 2011). As the target exhibits no evidence of mid-infrared excess, the spectrum was scaled to the photospheric model

\footnotetext{
12 The Cornell Atlas of Spitzer/IRS Sources (CASSIS) is a product of the Infrared Science Center at Cornell University, supported by NASA and JPL.
} 
using a least-squares fit weighted by the measurement uncertainties at wavelengths $<15 \mu \mathrm{m}$. Target fluxes were then extracted in two windows centered at 13 and $31 \mu \mathrm{m}$, and $4 \mu \mathrm{m}$ in width using the error-weighted average of values within the bin.

At far-infrared wavelengths, we combine ISO/ISOPHOT measurements at 60 and $90 \mu \mathrm{m}$ (Spangler et al. 2001) and a Spitzer/MIPS measurement at $70 \mu \mathrm{m}$ (Carpenter et al. 2009) with Herschel/PACS measurements at 70, 100, and $160 \mu \mathrm{m}$. The spatial resolution of $I S O$ is much poorer (1.5 beam FWHM) than that of Spitzer or Herschel $\left(6^{\prime \prime}-18^{\prime \prime}\right.$ beam FWHM); inclusion of background contamination and errors in the zero-level calibration elevating the $I S O$-measured fluxes is a possibility (del Burgo et al. 2003; Héraudeau et al. 2003). However, the target is well isolated in the Herschel maps, and there is good agreement among the measurements by all three facilities at similar wavelengths so we therefore do not consider it to have a significant impact on the shape of the SED.

The Herschel ${ }^{13}$ observations were first presented in Donaldson et al. (2012). Here we re-reduced these data to obtain revised values for the source fluxes based on updates to the instrument calibration made available in the intervening time. The data reduction and analysis were carried out in the same manner as described in Eiroa et al. (2013). The PACS data were reduced in the Herschel Interactive Processing Environment $^{14}$ (HIPE; Ott 2010) using the standard pipeline processing scripts (HIPE version 15, PACS calibration 78). Target fluxes were measured in each mosaic using an aperture of $15^{\prime \prime}$ radius and a sky annulus of $30^{\prime \prime}-40^{\prime \prime}$. Appropriate aperture and color corrections (determined by a blackbody fit to the aperture-corrected measurements), based on values tabulated in Balog et al. (2014), were applied to the measurements before modeling.

We also present submillimeter measurements taken from Herschel/SPIRE (program ot2_aroberge_3, PI: A. Roberge) and an APEX/LABOCA measurement at $880 \mu \mathrm{m}$ (Nilsson et al. 2010). The SPIRE observations were again reduced in HIPE version 15, using SPIRE calibration 14_3. The target fluxes in the three SPIRE maps were measured using the sourceExtractorSussextractor routine. The millimeter SED is further constrained by ALMA photometry at $1300 \mu \mathrm{m}$ (program 2012.1.00437.S, PI: D. Rodriguez) and an ATCA measurement at $9 \mathrm{~mm}$ taken from Marshall et al. (2017).

\section{Methodology and Results}

Here we undertake a determination of the stellar parameters by fitting a grid of stellar atmosphere models to archival highresolution spectra. We also inferred the same stellar parameters as well as the mass and age from stellar evolution models combined with a Bayesian approach. We then present a selfconsistent analysis of both the millimeter and scattered light imaging data to determine the disk architecture and dust albedo, respectively. The disk structure is applied as a constraint in the modeling of the disk SED in combination with photometry from archival sources.

\footnotetext{
${ }^{13}$ Herschel is an ESA space observatory with science instruments provided by European-led Principal Investigator consortia and with important participation from NASA.

${ }^{14}$ HIPE is a joint development by the Herschel Science Ground Segment Consortium, consisting of ESA, the NASA Herschel Science Center, and the HIFI, PACS and SPIRE consortia.
}

Table 2

Summary of Stellar Parameters

\begin{tabular}{lcc}
\hline \hline Parameter & Value & References \\
\hline R.A. $(\mathrm{hms})$ & 000552.54 & 1 \\
Decl. $(\mathrm{dms})$ & -414511.0 & 1 \\
Proper motions $\left(\mathrm{mas} \mathrm{yr}^{-1}\right)$ & $97.96,-76.51$ & 2 \\
Distance (pc) & $38.85 \pm 0.08$ & 2 \\
$V$ (mag) & $7.513 \pm 0.005$ & 3 \\
$B-V(\mathrm{mag})$ & $0.600 \pm 0.003$ & 3 \\
Spectral type & $\mathrm{G} 0$ & 4 \\
Luminosity $\left(L_{\odot}\right)$ & $1.216 \pm 0.005$ & 4 \\
Radius $\left(R_{\odot}\right)$ & $1.009 \pm 0.003$ & 4 \\
Mass $\left(M_{\odot}\right)$ & $1.116 \pm 0.012$ & 4 \\
Temperature $(\mathrm{K})$ & $6034 \pm 8$ & 4 \\
Surface gravity, $\log g$ & $4.478 \pm 0.006$ & 4 \\
Metallicity, $[\mathrm{Fe} / \mathrm{H}]$ & $0.02 \pm 0.04$ & 5 \\
$\nu$ sin $i\left(\mathrm{~km} \mathrm{~s}{ }^{-1}\right)$ & $17.5 \pm 0.5$ & 4 \\
Age $(\mathrm{Myr})$ & $50 \pm 16$ & 4 \\
\hline
\end{tabular}

References. 1. van Leeuwen (2007); 2. Lindegren et al. (2016); Gaia Collaboration et al. (2016, 2018); 3. Mermilliod et al. (1997); 4. This work; 5. Tsantaki et al. (2014).

\subsection{Stellar Parameters}

A summary of the stellar properties used in this work is given in Table 2. The stellar position, distance (parallax), proper motions, and $G$-band magnitude $(G=7.3833 \pm 0.0007)$ are taken from the Gaia DR2 catalog (Gaia Collaboration et al. 2016, 2018; Lindegren et al. 2016). The stellar physical properties were derived by modeling of available high-resolution spectra along with optical and near-infrared photometry.

The stellar parameters of HD 105, i.e., luminosity, radius, effective temperature, surface gravity, age, and mass, were derived by using the absolute $G$ magnitude, parallax, $B-V$ color, and $[\mathrm{Fe} / \mathrm{H}]$ as input parameters using the Bayesian approach applied in del Burgo \& Allende Prieto (2016, 2018). We derived an age of $50 \pm 16 \mathrm{Myr}$, which is in agreement with that one more constrained assuming membership of Tucana-Horologium, of $30 \mathrm{Myr}$ (C. del Burgo et al. 2018, in preparation). The color $B-V=0.600 \pm 0.003$ is taken from Mermilliod et al. (1997), and the metallicity $[\mathrm{Fe} / \mathrm{H}]=$ $0.02 \pm 0.04$ is from Tsantaki et al. (2014). The observed $G$ magnitude was converted to absolute magnitude $M_{G}$ using the Gaia DR2 parallax of $\pi=25.75 \pm 0.06$ mas (Gaia Collaboration et al. 2018). We find that HD 105 is a pre-mainsequence star. In order to derive the stellar parameters, we downloaded and arranged a grid of PARSEC isochrones (version 1.2S, Bressan et al. 2012; Chen et al. 2014, 2015; Tang et al. 2014), with steps in age of 5\%, steps in mass of $0.1 \%$, and steps of $0.02 \mathrm{dex}$ in $[\mathrm{Fe} / \mathrm{H}]$. The lower and upper limits of these parameters are well beyond $6 \sigma$ around the solution; see Table 2. Synthetic photometry estimates were obtained using filter curves from Evans et al. (2018) for the Gaia $G$ band, and from Maíz Apellániz (2006) for the $B$ and $V$ bands. For a more detailed description, see del Burgo \& Allende Prieto (2018).

In addition, high-resolution FEROS and HARPS spectra were also used to obtain the stellar effective temperature $T_{\text {eff }}$, metallicity $[\mathrm{Fe} / \mathrm{H}]$, projected rotational velocity $\nu \sin i$, and surface gravity $\log g$. We performed a comparison with BTSettl stellar atmosphere models (Allard et al. 2012). The models in the grid were modified in order to be compared with the 
FEROS spectrum similar to the approach of del Burgo et al. (2009). First, the synthetic spectra were transformed to take into account the stellar projected rotational velocity $(\nu \sin i)$ using the formalism of Gray (1992), with a limb-darkening parameter equal to 0.8 . These spectra were convolved with a Gaussian that mimics the instrumental profile along the dispersion axis. The resulting spectra were rebinned to the same resolution and grid of the observed spectrum. This was corrected from the velocity shift from a cross-correlation analysis with the models. All modeled and observed spectra were normalized before performing the comparison.

We obtain values from the FEROS spectrum $(R=48,000)$ of $T_{\text {eff }}=6000 \pm 50 \mathrm{~K}, \log g=4.50 \pm 0.25$, and $\nu \sin i=$ $17.5 \pm 0.5 \mathrm{~km} \mathrm{~s}^{-1}$. Values derived from the higher resolution HARPS spectrum $(R=115,000)$ are consistent with those of FEROS, with the FEROS values being determined at higher signal-to-noise ratio. These values are consistent with those derived from the aforementioned stellar atmosphere models.

We have a good agreement between the stellar evolution analysis and the stellar atmosphere model fitting for the parameters in common. The values obtained through this analysis are likewise consistent with available values from the literature, e.g., Valenti \& Fischer (2005) and Tsantaki et al. (2014).

The $\mathrm{H} \alpha$ and $\mathrm{Ca}$ II $\mathrm{H} \& \mathrm{~K}$ lines in the FEROS spectrum are presented in Figure 1. The median FEROS spectrum, calculated from the available spectra (in black), is plotted together with our best-fitting model. Some regions of the $\mathrm{H} \alpha$ segment of the spectrum have been blanked out due to contamination from telluric lines at those wavelengths (see Figure 1, top). Despite the low $\nu \sin i$ (consistent with a more pole-on viewing angle), we see clear evidence of Ca II $\mathrm{H} \& \mathrm{~K}$ emission in the spectrum (see Figure 1, bottom). This is consistent with activity in the stellar chromosphere, an indicator of stellar youth.

We also examined the high-resolution spectra for evidence of gas revealed by the presence of circumstellar absorption or emission lines. The origin of such gas may be primordial or secondary from, e.g., photodesorption or cometary activity. We note that the FEROS fiber input has a 2 " footprint on the sky that could include light scattered from material in the circumstellar disk, depending on the seeing quality. No evidence of such features is seen. No evidence of gas features in either absorption (implying cool gas) or emission (implying hot gas) was identified in the spectra.

\subsection{ALMA Millimeter Imaging Data}

An ALMA band $6(1.3 \mathrm{~mm})$ observation for HD 105 was downloaded from the ESO ALMA Science Archive. ${ }^{15}$ The observation was originally carried out as part of project 2012.1.00437.S (PI: D. Rodriguez) during Cycle 1. The spectral setup consists of four windows. Three windows were set up to measure the continuum, each with 128 channels over a $2 \mathrm{GHz}$ bandwidth. The fourth covered the ${ }^{12} \mathrm{CO}(2-1)$ line at $230.538 \mathrm{GHz}$ and sampled its $0.94 \mathrm{GHz}$ bandwidth with 3840 channels $\left(0.32 \mathrm{~km} \mathrm{~s}^{-1}\right)$, providing a velocity resolution of $0.64 \mathrm{~km} \mathrm{~s}^{-1}$. In combination, the four channels provide a $6.9 \mathrm{GHz}$ bandwidth to study the continuum emission. The onsource integration time for HD 105 was $2238 \mathrm{~s}$. Neptune was used as the flux calibrator, J0006-0623 was the bandpass calibrator, and J0012-3954 was the phase calibrator.

\footnotetext{
15 http://almascience.eso.org/aq/
}
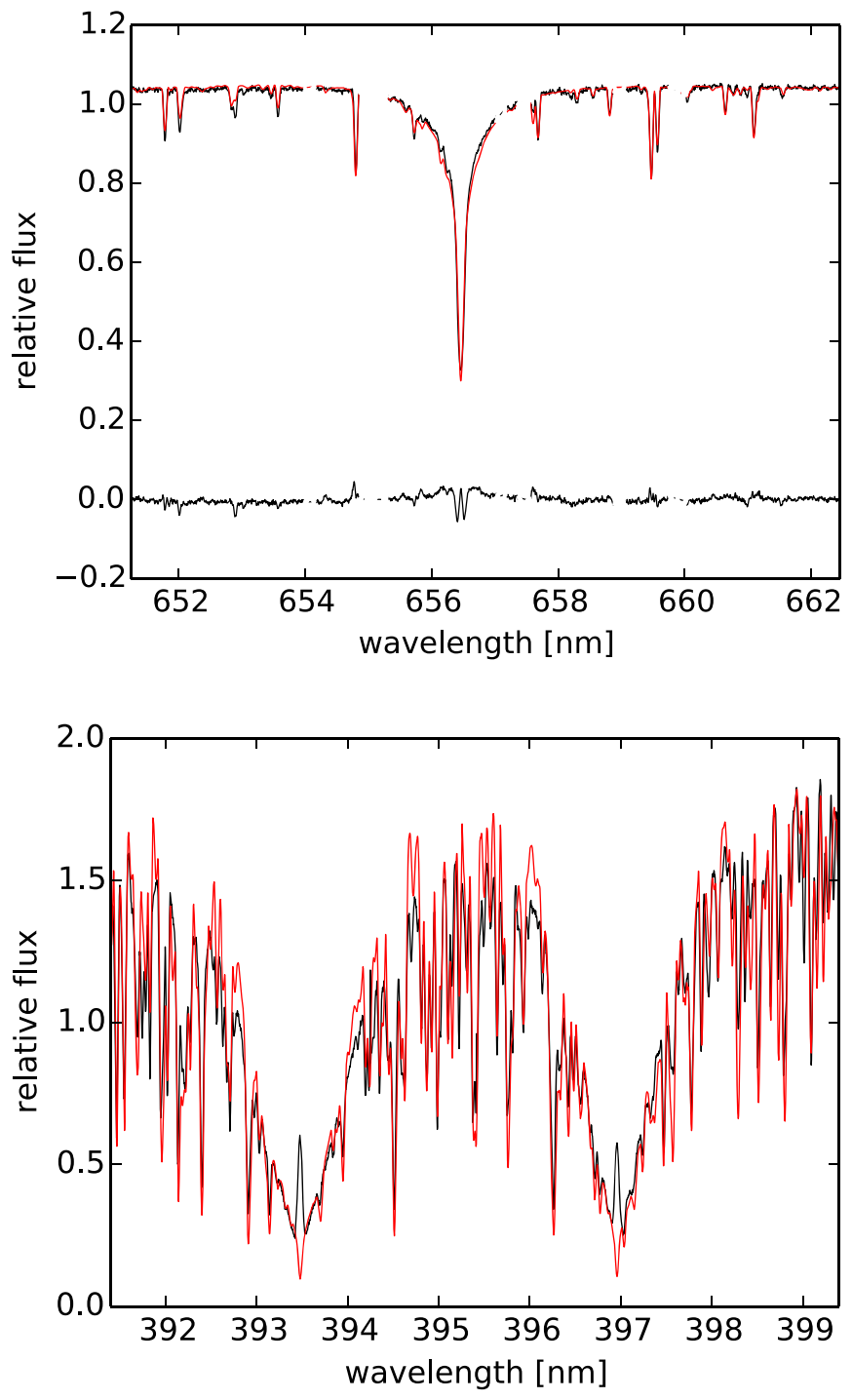

Figure 1. Segments of the FEROS spectrum covering the $\mathrm{H} \alpha$ (top) and $\mathrm{Ca}$ II H\&K (bottom). The median stellar spectrum from X FEROS observations is presented in black, while the best-fitting model is presented in red. Some regions have been blanked out due to contamination from telluric lines. The residual (observed - model) spectrum is shown for clarity in the $\mathrm{H} \alpha$ panel.

Calibration and reduction of the ALMA observation were carried out in CASA 4.1 using the provided scripts. Image reconstruction was carried out using the clean task, combining all four spectral windows for the greatest signal-to-noise ratio. We reconstruct the image using natural weighting. With natural weighting, the continuum image r.m.s. noise is $26.8 \mu \mathrm{Jy}$ beam $^{-1}$. The dirty beam has an ellipsoidal FWHM 0 ". $95 \times 0$ ". 67 at a position angle of $88^{\circ}$, equivalent to a spatial resolution of $34 \times 26$ au.

\subsubsection{Disk Architecture}

The disk is visible as an annular structure centered on the stellar position, but the low signal-to-noise ratio of the observations means that it is not detected with high significance $(>3 \sigma)$ at all position angles. The ALMA image and the best-fit annular disk model are presented in Figure 2.

We model the architecture of HD 105's disk as a single annulus with a semimajor axis $R$ and width $\Delta R$ oriented at a 

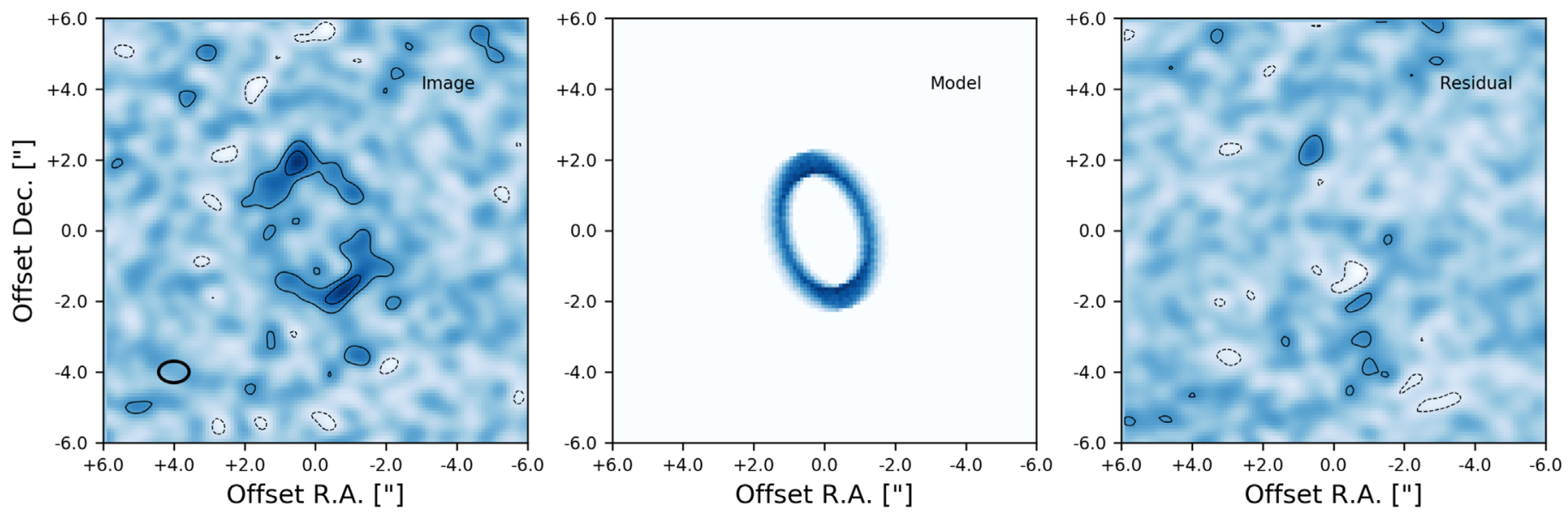

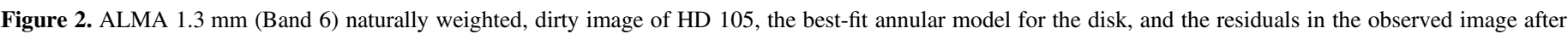

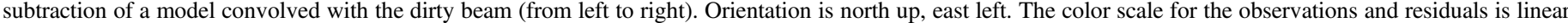

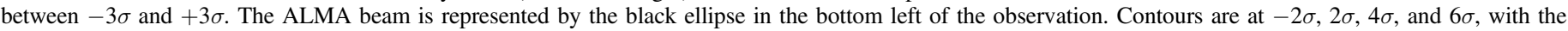
broken contour denoting negative values.

position angle $\theta$ and inclination $i$. The disk surface brightness exponent $\alpha$ is assumed to be constant, and a vertical opening angle of $10^{\circ}$, similar to the solar system, was assumed. A grid of models is generated spanning reasonable values for each of these parameters. To determine the best-fit properties for the disk, we subtract each disk model, convolved with the dirty ALMA beam (FWHM $\sim 0$ " $9 \times 0$ ".7), from the observed image and seek to minimize the residuals within the region of the model-subtracted image where there is significant emission in the observed image.

The best-fit architecture derived from the model fitting is dominated by the disk ansae, which are the only regions of the disk detected at a high signal-to-noise ratio $(>3 \sigma)$. The disk semimajor axis is 2 !" $16 \pm 0$ "' 13 (85 $\pm 5 \mathrm{au}$ ), with an inclination of $50^{\circ} \pm 5^{\circ}$ with respect to the line of sight at a position angle of $15^{\circ} \pm 5^{\circ}$. The width of the disk annulus is 0 "'75 \pm 0 !" $25(30 \pm 10 \mathrm{au})$. The belt is perhaps marginally resolved in the ALMA image, but the quality of the data does not allow us to conclude that, so we hereafter assume the belt is unresolved. The best-fit disk architecture obtained from this approach is summarized in Table 3.

The total flux of the disk in the submillimeter, $1.45 \pm 0.28 \mathrm{mJy}$, may be used to calculate the mass of dust grains, assuming that the disk is fully optically thin at $1.3 \mathrm{~mm}$. For a disk observed at a frequency $\nu$ with a flux $F_{\nu}$, the dust mass, $M_{\text {dust }}$, is given by

$$
M_{\text {dust }}=F_{\nu} d_{\star}^{2} / \kappa_{\nu} B_{\nu, T_{\text {dust }}},
$$

where $d_{\star}$ is the stellar distance, $\kappa_{\nu}$ is the dust opacity, $T_{\text {dust }}$ is the dust temperature, and $B_{\nu, T_{\text {dust }}}$ is the Planck function (Zuckerman \& Becklin 1993; Draine 2006). Assuming the dust opacity $\kappa$ is $1.7 \mathrm{~g} \mathrm{~cm}^{-2}$ (Beckwith et al. 1990; Pollack et al. 1994; Draine 2006), which is commonly assumed for debris dust (e.g., Panić et al. 2013; Holland et al. 2017), we calculate a mass of $0.035_{-0.009}^{+0.013} M_{\oplus}$ for the dust. This value does not include uncertainty on the parameter $\kappa$, which leads to an uncertainty in $M_{\text {dust }}$ of a factor 3-5.
Table 3

Architecture of HD 105's Disk Determined from the ALMA Image

\begin{tabular}{lcclc}
\hline \hline Parameter & Range & Values & Spacing & Value \\
\hline Inclination $\left(^{\circ}\right)$ & $0-90$ & 19 & Linear & $50 \pm 5$ \\
Position Angle $\left(^{\circ}\right)$ & $0-90$ & 19 & Linear & $15 \pm 5$ \\
Semimajor axis (au) & $50-150$ & 41 & Linear & $85 \pm 5$ \\
Semimajor axis $\left({ }^{\prime \prime}\right)$ & & & & $2.16 \pm 0.13$ \\
Belt width (au) & $10-50$ & 21 & Linear & $30 \pm 10$ \\
Belt width (") & & & & $0.75 \pm 0.25$ \\
S.B. exponent, $\alpha$ & 0.0 & 1 & Fixed & 0.0 \\
Total disk flux (mJy) & $1.0-4.0$ & 301 & Linear & $1.45 \pm 0.28$ \\
Dust mass $\left(M_{\oplus}\right)$ & & & & $0.035_{-0.009}^{+0.013}$ \\
\hline
\end{tabular}

\subsubsection{Non-detection of Cold CO Emission}

Molecular carbon monoxide $(\mathrm{CO})$ has been identified in around a dozen debris-disk systems (e.g., Greaves et al. 2016; Moór et al. 2017), believed in most cases to be the result of secondary production after liberation from exocomets (Kral et al. 2017). Most of the stars identified with gas in their debris disks are young $\left(t_{\text {age }}<30 \mathrm{Myr}\right)$ A-type stars, with relatively bright debris disks $\left(L_{d} / L_{\star} \sim 10^{-3}\right)$. Here we search the ALMA observation for emission from the disk associated with the $\mathrm{CO}(2-1)$ transition at $230.538 \mathrm{GHz}$ following the method employed to extract $\mathrm{CO}$ emission at low signal-to-noise ratio in ALMA data as presented in Matrà et al. (2015) and Marino et al. (2016, 2017).

To begin, we produced a continuum-subtracted measurement set from the HD 105 data using uvcontsub. The continuum-subtracted data set is then "clean"ed to produce an image cube with the same weighting and pixel scale as the continuum image. The cube spans barycentric velocities between -25 and $25 \mathrm{~km} \mathrm{~s}^{-1}$, with a step size of $0.5 \mathrm{~km} \mathrm{~s}^{-1}$. The cube is corrected for the effect of the primary beam using the impbcor task before further analysis. No significant $\mathrm{CO}$ emission is observed in the velocity-integrated, continuumsubtracted image produced by integrating the cube.

We then proceed by implementing the spectro-spatial filtering technique presented in Matrà et al. (2017). Any circumstellar $\mathrm{CO}$ is assumed to originate from collisions between planetesimals and therefore reside in the same region 
around the star as the debris dust detected in the continuum image. The CO is further assumed to be situated in a vertically flat disk, orbiting with Keplerian velocity around a star of $1.114 M_{\odot}$. We then determine the projected radial velocity for each position in the disk image given the extent and inclination of the disk for cases of the disk rotating toward or away from us. Using the predicted velocity field, the spectrum at each position is then shifted to match the stellar velocity (i.e., spectral filtering). We then integrate over all positions where continuum emission is detected to avoid contributions to the $\mathrm{CO}$ spectrum from locations and velocities where no circumstellar $\mathrm{CO}$ is expected (i.e., spatial filtering). The spectro-spatial filtering yields a $3 \sigma$ upper limit of $8.5 \mathrm{mJy} \mathrm{km} \mathrm{s}^{-1}$ to $\mathrm{CO}$ emission.

We use a non-local thermodynamic equilibrium $\mathrm{CO}$ excitation code to calculate a $\mathrm{CO}$ mass upper limit, following Matrà et al. (2018b). This code includes fluorescent excitation from the central star at ultraviolet wavelengths. Depending on the excitation conditions, we determine a $\mathrm{CO}$ mass upper limit ranging from $2.5 \times 10^{-7}$ to $2.5 \times 10^{-6} M_{\oplus}$. This translates to upper limits on the $\mathrm{CO}$ and $\mathrm{CO}_{2}$ fraction (by mass) in exocomets between $<88 \%$ and $<46 \%$ for the respective mass upper limits. This limit is consistent with the range of values for other exocometary disks and solar system bodies (see e.g., Matrà et al. 2017).

\subsection{Detection of Scattered Light}

HD 105 has been observed by both HST/NICMOS and VLT/SPHERE. The disk was not directly visible in either data set. However, we recover the disk at the correct angular separation and orientation using an angular averaging technique to aggregate the disk emission to a detectable level. From this measurement of the disk-scattered light brightness, we determine the albedo of the dust grains at near-infrared wavelengths.

\subsubsection{HST/NICMOS}

HD 105 was observed with the $H S T /$ NICMOS instrument at two epochs using the coronagraphic mode (mask radius 0 ". 3 ) of the mid-resolution NIC2 channel $\left(0\right.$ "'07565 pixel $\left.^{-1}\right)$. The first data were acquired on UT-1998-11-03 as part of program GTO-7226 (PI: E. Becklin), a survey looking for giant planets around young nearby stars (Lowrance et al. 2005), using the F160W filter of the bandpass very comparable to the $H$ band (pivot wavelength $1.600 \mu \mathrm{m}, 98 \%$ integrated bandwidth $0.410 \mu \mathrm{m})$. The second data set was obtained on UT-2006 July 23 as part of program GO-10527 (PI: D. Hines), a survey looking for debris disks around stars with infrared excess detected with Spitzer as part of the FEPS program (Hillenbrand et al. 2008). The data were obtained with the F110W filter, which is twice as extended toward shorter wavelengths as the $J$ band (pivot wavelength $1.116 \mu \mathrm{m}, 98 \%$ integrated bandwidth $0.584 \mu \mathrm{m})$.

The F160W data were acquired at two different spacecraft orientations, obtaining a total of six exposures with HST rolled by $\sim 30^{\circ}$ in the middle of the sequence, to enable subtraction of the PSF with roll differential imaging (Lowrance et al. 1999). The total exposure time is $1344 \mathrm{~s}$ for this data set. The F110W data set includes seven exposures all obtained with the same telescope orientation, for a total exposure time of $2016 \mathrm{~s}$.

We reprocessed both data sets as part of the Archival Legacy Investigations for Circumstellar Environments (ALICE) program (PI: R. Soummer), a consistent reanalysis of the NICMOS coronagraphic archive using modern PSF subtraction techniques (Choquet et al. 2014; Hagan et al. 2018). Assembling PSF libraries from multiple reference stars in the whole archive and using the PCA-based KLIP algorithm for PSF subtraction (Soummer et al. 2012), this program demonstrated a gain in point-source sensitivity by a factor of 20 at $1^{\prime \prime}$ from the star over classical reference star differential imaging (Debes et al. 2016) and enabled the detection of 11 faint debris disks from NICMOS archival data (Soummer et al. 2014; Choquet et al. 2016, 2017, 2018).

For HD 105, we used the reprocessed data from the ALICE public database $^{16}$ for the F110W data set and an unpublished ALICE reduction for the F160W data set with an $11^{\prime \prime} \times 11^{\prime \prime}$ field of view, which is more favorable to disk detection with sensitivity limits better than the data published in the ALICE database. PSF subtraction for the F160W data set was achieved using the 69 first eigenmodes of a library assembling 125 images from reference stars exclusively and excluded a central zone within a radius of 12 pixels. The F110W data set was PSF-subtracted with the 211 first eigenmodes of a 640 image library from reference stars only, excluding a central area of radius 5 pixels. The final images were obtained by rotating all exposures to north pointing up, co-adding and scaling them to surface brightness units based on NIC2's plate scale and its calibrated photometric factors $\left(F_{\nu}=1.21121 \mu \mathrm{Jy} \mathrm{s}^{-1}\right.$ in $\mathrm{F} 110 \mathrm{~W}, F_{\nu}=1.49585 \mu \mathrm{Jy} \mathrm{s} \mathrm{DN}^{-1}$ in $\left.\mathrm{F} 160 \mathrm{~W}\right)$. We are able to recover the disk-scattered light in the F160W filter image in the disk's ansae at low signal-to-noise ratio, as shown in Figure 3.

To obtain a more significant measurement of the disk surface brightness than can be obtained from the reduced images, we make use of the high spatial resolution of the scattered light image and measure the surface brightness radial profiles. We computed the surface brightness averaged in 5 pixel wide $(0$ ". 38) elliptical apertures of increasing semimajor axes $a$ with semimajor axis $a$ increasing in steps of 1 pixel (76 mas) and semiminor axis $b=a \cos \left(50^{\circ}\right)$, oriented $15^{\circ}$ east of north, to trace the disk deprojected average radial profile according to the ALMA detection geometry. We estimate the corresponding uncertainties assuming uncorrelated noise, as $\left.\sigma / \sqrt{(} N_{\text {pix }}\right)$ with $\sigma$ the standard deviation in each aperture and $N_{\text {pix }}$ the number of pixels in the aperture. Since only reference stars were used for PSF subtraction, these measurements are not affected by disk self-subtraction. They are, however, subject to oversubtraction by the PCA algorithm along with PSF features (Soummer et al. 2012; Pueyo 2016). We corrected our average profiles and uncertainties by estimating the algorithm throughput with analytical forward modeling. From this exercise, we find a significant peak with a surface brightness of $15 \pm 1 \mu \mathrm{Jy} / \operatorname{arcsec}^{2}$ at a separation of around 2 !" 2 from the star, consistent with the ALMA image. The binned radial profiles are presented in Figure 3.

\subsubsection{VLT/SPHERE}

HD 105 was observed with the Spectro-Polarimeter Highcontrast Exoplanet REsearch (SPHERE; Beuzit et al. 2008) on the night of 2015 October 2. These observations used the InfraRed Dual-beam Imager and Spectrograph (IRDIS; Dohlen et al. 2008) of SPHERE to obtain high-contrast and high-angularresolution images of the circumstellar environment of HD 105,

\footnotetext{
${ }^{16}$ https://archive.stsci.edu/prepds/alice/
} 

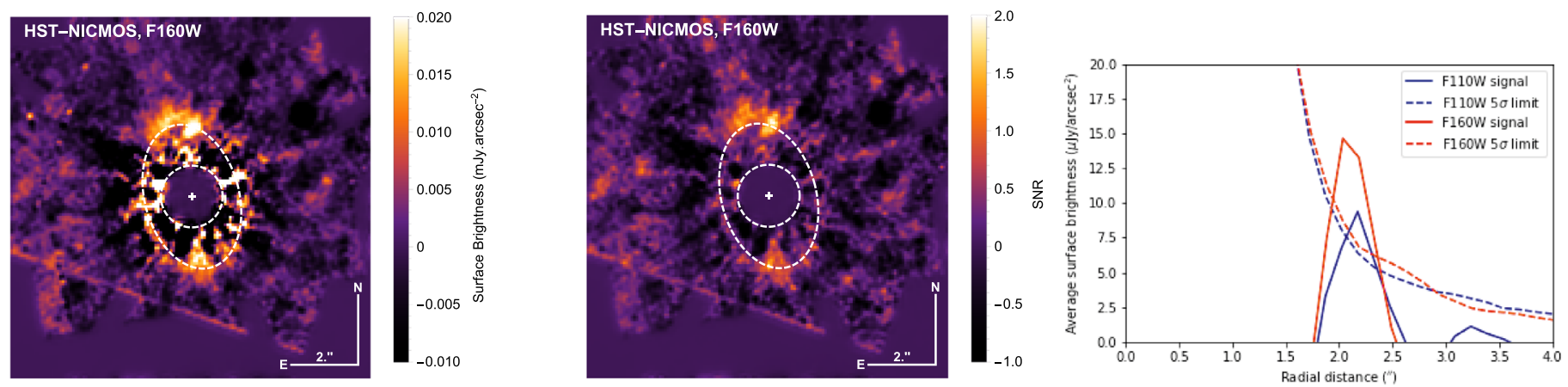

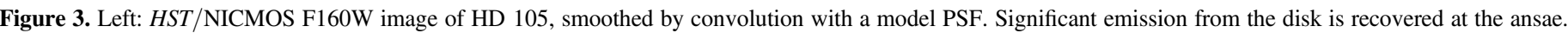

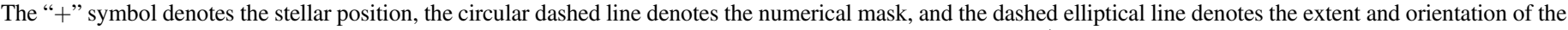

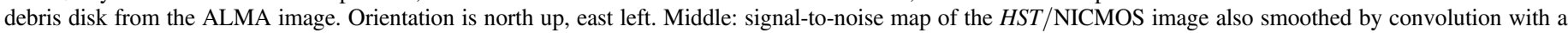

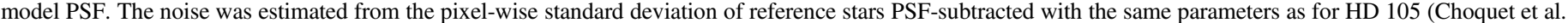

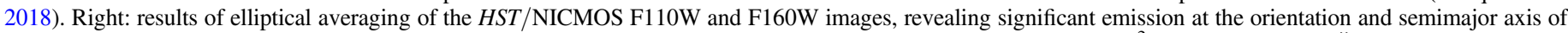

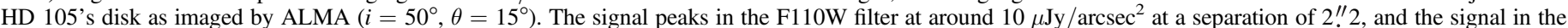

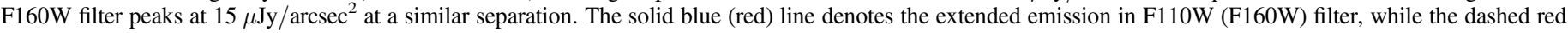
(blue) line denotes the $5 \sigma$ surface brightness limit in the respective filters.

in the broadband $H$ filter (centered at $1.625 \mu \mathrm{m}$, width $291 \mathrm{~nm}$ ) with the apodized Lyot coronagraph of diameter 185 mas. They are part of the SPHERE High-Angular Resolution Debris Disk Survey ${ }^{17}$ (SHARDDS; J. Milli et al. 2018, in preparation). SHARDDS is an open-time program on SPHERE to perform the first comprehensive near-infrared survey of all bright, nearby debris disks (infrared excess greater than $10^{-4}$ ), yet undetected in scattered light, within $100 \mathrm{pc}$. It already led to detections of several disks and a brown dwarf companion: HD 114082 (Wahhaj et al. 2016), 49 Ceti (Choquet et al. 2017), and HD 206893 (Milli et al. 2017a). HD 105 was observed for a total of 50 minutes on-source, among which the total exposure time is $2432 \mathrm{~s}$, in pupil-stabilized mode to allow Angular Differential Imaging (Marois et al. 2006). The source was observed just before meridian crossing, which resulted in a parallactic angle rotation of $35^{\circ}$.

The data were sky-subtracted, flat-fielded, and bad-pixelcorrected using the official SPHERE Data Reduction and Handling pipeline (Pavlov et al. 2008) in order to make a temporal cube of 606 frames. Each coronagraphic frame was re-centered using the set of four satellite spots imprinted in the image during the centering sequence. This sequence is obtained by applying a waffle pattern to the deformable mirror and was done prior to and after the deep science coronagraphic observations. Then, different data reduction techniques were applied to subtract the stellar halo and to try to detect the scattered light of the disk.

At the expected semimajor axis of the disk of 2 !" 16 , the technique that yields the best sensitivity for point-source detection is the classical Angular Differential Imaging (cADI; Marois et al. 2006) technique. However for an azimuthally extended structure such as the HD 105 disk, the throughput of the algorithm is very low because of self-subtraction (Milli et al. 2012), and we estimated it to be a few percent. The $5 \sigma$ sensitivity of the ADI reduction is $100 \mu \mathrm{Jy}$ at 2 !' 2 after throughput correction, and we are therefore unable to confirm the HST/NICMOS detection with this data reduction technique.

Therefore, we also applied the Reference Star Differential Imaging (R[S]DI) technique, commonly used in space-based observations (Schneider et al. 1999; Lafrenière et al. 2009;

${ }^{17}$ ESO program ID 096.C-0388(A).
Soummer et al. 2011), and more recently applied to groundbased observations (Gerard \& Marois 2016). We implemented this technique in the SHARDDS program by building a library of all the coronagraphic images obtained in the program. We then selected 610 images from the 22 stars most correlated with the ones from HD 105 (excluding the HD 105 images themselves) and used this set of references to subtract the stellar halo, using a custom PCA algorithm, keeping 100 modes. The disk is not directly visible in the VLT/SPHERE image.

The image is binned by a factor of 4 , giving a new pixel size of 49 mas, corresponding to the measured FWHM in the noncoronagraphic image. In the image reduced with PCA-RDI, the flux is integrated in elliptical apertures having an aspect ratio matching the inclination of the disk as seen by ALMA, and a radial width of 10 binned pixels or 490 mas. This ellipse is increased iteratively by steps of 1 binned pixel or 49 mas, and at each iteration, the signal is computed as the sum of the flux over this elliptical aperture. It is corrected for the PCA throughput and converted into $\mu \mathrm{Jy} / \operatorname{arcsec}^{2}$ using the binned pixel size of 49 mas and the star flux as measured in the noncoronagraphic image. The noise is given by the standard deviation of the pixels over the same elliptical annulus.

This averaging technique increases the depth to which we can detect disk-scattered light emission using the geometrical prior from the resolved millimeter detection. We find a peak in emission at $5.9 \mu \mathrm{Jy} / \operatorname{arcsec}^{2}$ for a semimajor axis of $2 ! 3$, consistent with the disk semimajor axis, as shown in Figure 4. This is a factor of $\sim 4$ fainter than the disk detected by $H S T$ / NICMOS; examination of the separate channels reveals that the right channel is consistent with the NICMOS-detected signal in both semimajor axis and brightness, but the left channel is not. Both channels are consistent in the presence of emission between $1 . " 5$ and 3 "'5, consistent with HD 105's disk. The origin of the discrepancy between the HST/NICMOS and VLT/SPHERE detections potentially lies in the estimation of noise, which has been assumed Gaussian here, but it can be seen in the reduced image that the noise is non-Gaussian.

\subsubsection{Combined Modeling}

The scattered light measurements from HST/NICMOS and VLT/SPHERE can be used to calculate the albedo of the dust 

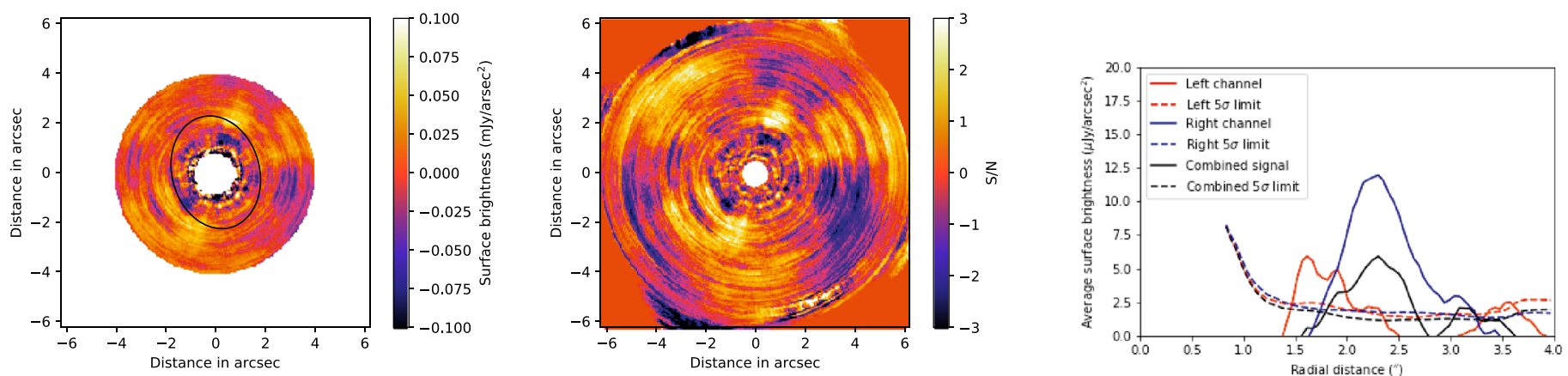

Figure 4. Left: VLT/SPHERE PCA-RDI reduced image of HD 105. The black ellipse denotes the location of the disk in the ALMA image. Middle: signal-to-noise map of the VLT/SPHERE image, combining both left and right detector channels. Right: results of elliptical annular averaging of the VLT/SPHERE image, revealing significant emission at the orientation and semimajor axis of HD 105's disk as imaged by ALMA $\left(i=50^{\circ}, \theta=15^{\circ}\right)$. The emission peaks at $5.9 \mu \mathrm{Jy} / \operatorname{arcsec}^{2}$ at a separation of 2 ." 3 , denoted by the black solid line in the figure; the black dashed line denotes the $5 \sigma$ surface brightness limit. The red and blue lines denote the measured brightness and $5 \sigma$ limits for the left and right channels, respectively. We note that the two channels are very different, but are consistent in that they reveal significant emission between $1 " .5$ to 3 ". 0 from the star on the same orientation as the disk in the ALMA image. See text for details.

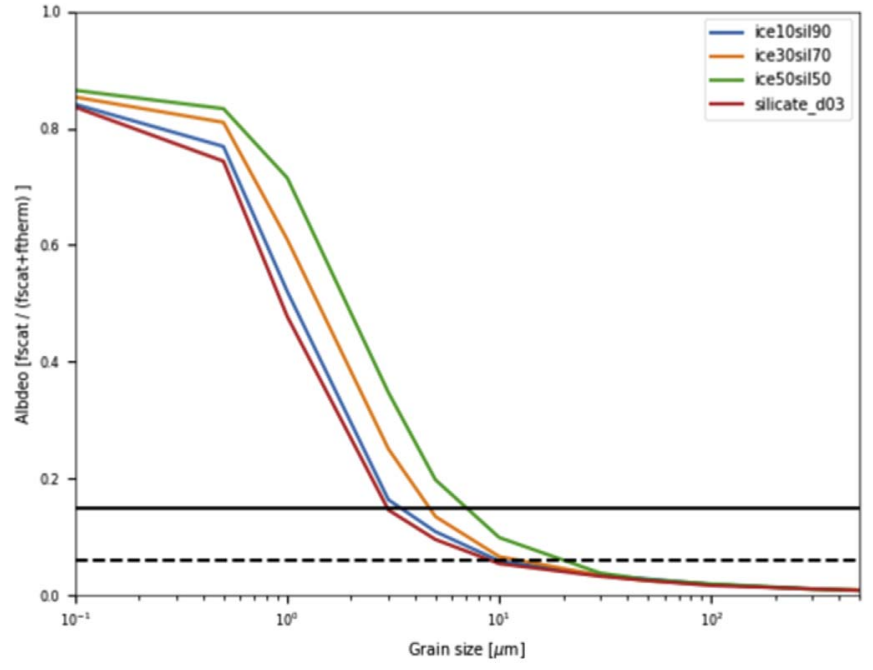

Figure 5. Plot of dust grain size vs. scattering albedo illustrating the constraints on dust grain size and composition possible with the new scattered light detection of HD 105's disk. The horizontal black lines denote the expected dust albedo required to reproduce the mean surface brightness from NICMOS (solid, $\omega=0.15$ ) and SPHERE (dashed, $\omega=0.06$ ). The colored lines denote the scattering albedos for disks composed of dust grains with a single size and composition. We find that silicate (or slightly icy) dust grains around a few $\mu \mathrm{m}$ in size are consistent with the NICMOS-derived scattering albedo; larger grains $(\geqslant 10 \mu \mathrm{m})$ are required to reproduce the SPHERE-derived albedo, but this would be inconsistent with the typical grain size of dust around Sun-like stars of a few to 10 times the blow-out radius.

grains. Assuming optically thin dust and using the approximation from Weinberger et al. (1999), the maximum allowed optical depth $\tau$ times the albedo $\omega$ is $4 \pi \phi^{2} S / F$, where $S$ is the surface brightness of the disk in $\mathrm{mJy} / \operatorname{arcsec}^{2}, F$ the total star flux in $\mathrm{mJy}$, and $\phi$ the separation of the scatterers. Approximating the optical depth by $\tau=2 f \phi \cos (i) /[d \phi(1-\omega)]$, where $d \phi$ is the disk width in arcsec and $f$ the fractional luminosity, we obtain

$$
S=\frac{f F \omega}{(2 \pi \times \phi \times d \phi \times \cos (i))(1-\omega)} .
$$

Using a disk semimajor axis $\phi=2$ ". 16 with a width $d \phi=0$ !" 75 at an inclination $i=50^{\circ}$, we obtain an albedo of 0.15 based on the NICMOS surface brightness $\left(15 \mu \mathrm{Jy} / \operatorname{arcsec}^{2}\right)$, or 0.06 based on the SPHERE measurement $\left(5.9 \mu \mathrm{Jy} / \operatorname{arcsec}^{2}\right)$. The apparent surface brightness can be significantly enhanced in case of strong forward scattering (Hedman \& Stark 2015; Olofsson et al. 2016; Milli et al. 2017b) but given the inclination of the HD 105 disk, scattering angles below $40^{\circ}$ are not probed.

With the radial averaging, we have been able to measure dust albedos a factor of 3-10 deeper than expected. The detection of HD 105 in scattered light made here points the way to recovery of similarly faint disks in scattered light where the disk geometry is already known from spatially resolved continuum imaging. Deeper near-infrared or optical images are required to spatially resolve the scattered light disk, in particular to determine the degree of asymmetry in the dust scattering.

\subsection{Constraints on the Dust Composition}

Using the mean surface brightness from the scattered light detections, we can use the inferred albedos to constrain the dust grain composition. We calculate the scattering albedo $\omega=F_{\text {scat }} /\left(F_{\text {scat }}+F_{\text {therm }}\right)$, where $F_{\text {scat }}$ is the scattered light fractional luminosity in the F160W filter, and $F_{\text {therm }}$ is the dust continuum fractional luminosity. Following Choquet et al. (2018), we assume that the debris disk to be composed entirely of dust grains with a single size and of a single composition. It is further assumed that the same dust grains responsible for the scattered light are also responsible for the thermal emission so the value of $F_{\text {therm }}$ is therefore held fixed for all the tested combinations of grain size and compositions, i.e., $L_{\text {dust }} / L_{\star}=$ $2.6 \times 10^{-4}$. For consistency, we keep the dust mass of the disk fixed to that calculated for HD 105 based on its ALMA millimeter flux.

For each combination of grain size and composition, we calculate the scattered light brightness of the disk at the observed orientation and inclination of HD 105, convolving the disk's scattered light SED with the F160W bandpass to determine the scattered light fractional brightness $F_{\text {scat }}$ and hence the scattering albedo $\omega$. The scattered light modeling is carried out using the 3D radiative transfer code HYPERION (Robitaille 2011). We test grain sizes from 0.1 to $500 \mu \mathrm{m}$, and dust compositions of pure astronomical silicate and water ice: astronomical silicate mixtures in the ratios 10:90, 30:70, and 50:50. Optical constants for the materials were taken from Draine (2003; astronomical silicate) and Li \& Greenberg (1998; water ice). Calculation of the optical constants for composite materials was done using the Bruggeman effective medium theory (Bruggeman 1935). 


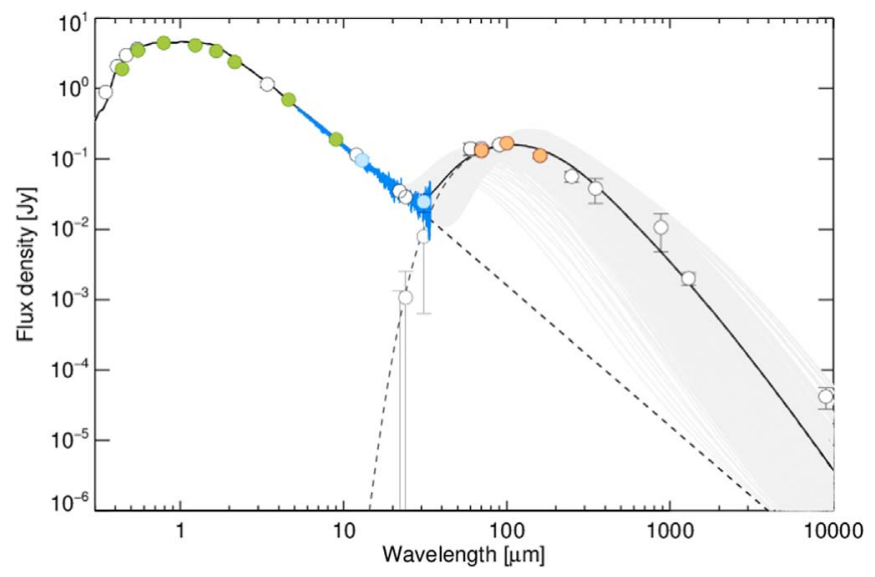

Figure 6. SED of HD 105, from radiative transfer modeling. The green dots denote the optical and infrared photometry used to scale the stellar photosphere model. The blue lines and dots denote Spitzer/IRS data. The orange dots denote Herschel/PACS data. The white dots denote ancilliary photometry from various literature sources (see Table 1). Uncertainties are $1 \sigma$. The dark gray data points denote excess values. The grayed-out region denotes the envelope of disk models produced from the grid parameter space. The dashed line denotes the individual stellar photosphere and disk components, while the solid line is the total emission.

We present the results of the scattering albedo analysis in Figure 5. This illustrates that dust grain sizes of a few microns and either pure silicate or moderately icy compositions (3-20 $\mu \mathrm{m}$, depending on the assumed composition and dust albedo), are consistent with the dust albedo derived from the NICMOSobserved mean disk surface brightness.

\subsection{Revised SED Model}

Here we combine the results previously determined from each data set in order to model the full system. We use the disk architecture, as determined from the ALMA image, and the dust scattering albedo measurement from the scattered light images, as constraints in a radiative transfer model of the debris disk. We model the disk as being an annulus lying 70 to $100 \mathrm{au}$ from the star with uniform radial surface brightness, as per the ALMA image. The disk is composed of compact, spherical dust grains described by a power-law size distribution $\left(d n \propto a^{-q} d a\right)$ with exponent $q$ spanning a size range from $a_{\min }$ to $a_{\max }$. The minimum dust grain size is a free parameter, but the maximum grain size is fixed at $10 \mathrm{~mm}$ to best replicate the millimeter wavelength photometry. The dust composition is assumed to be pure astronomical silicate (Draine 2003).

The resultant disk SED based on the best-fit model parameters is presented in Figure 6, and a summary of the best-fit disk properties (including the disk extent as fixed input) is provided in Table 4. The stellar photosphere component (dashed black line) is represented by a Castelli-Kurucz model (Castelli \& Kurucz 2004) appropriate to the stellar spectral type $\left(\log g=4.5 \mathrm{~cm} \mathrm{~s}^{-2}, T_{\text {eff }}=6000 \mathrm{~K},[\mathrm{Fe} / \mathrm{H}]=0.02\right)$ scaled to the optical and near-infrared fluxes (green data points). The blue and red data points denote the Spitzer/IRS and Herschel/ PACS fluxes, respectively. The gray data points are the excess (i.e., total - star) fluxes for wavelengths $>20 \mu \mathrm{m}$. The individual models comprising the model grid used to fit the observations are represented by the set of solid light gray lines, with the best-fit disk model denoted by the dashed dark gray
Table 4

Modeling Results from the Power-law fit to the Disk Emission

\begin{tabular}{lcclc}
\hline \hline Parameter & Range & Values & Spacing & Value \\
\hline Semimajor axis (au) & & 1 & Fixed & 85 \\
Belt width (au) & 0.0 & 1 & Fixed & 30 \\
$\alpha$ & $0.5-20.5$ & 41 & Linear & $3.25_{-1.00}^{+3.00}$ \\
\hline$a_{\min }(\mu \mathrm{m})$ & $10^{4}$ & 1 & Fixed & $10^{4}$ \\
$a_{\max }(\mu \mathrm{m})$ & $2.50-4.50$ & 11 & Linear & $3.55_{-0.15}^{+0.30}$ \\
$q$ & $\ldots$ & 1 & $\ldots$ & Astro. sil. \\
\hline Composition & & & &
\end{tabular}

line. The total model of the system (star+disk) is denoted by the solid black line.

\section{Discussion}

There is a growing body of work examining the resolved extent of debris disks as a function of their host stars' luminosities (e.g., Booth et al. 2013; Morales et al. 2013; Pawellek et al. 2014; Pawellek \& Krivov 2015). Generally, the resolved extent of debris disks around lower luminosity stars is found to be greater than what would be predicted from their blackbody temperature when compared to debris disks around higher luminosity stars.

We calculate the parameter $\Gamma\left(R_{\text {disk }} / R_{\mathrm{bb}}\right)$ for HD 105 's disk using the relationship between stellar luminosity and disk temperature (radius) derived in Pawellek \& Krivov (2015), tacitly assuming a dust composition of ice and astronomical silicate. We find $\Gamma=2.43 \pm 0.42$, whereas a value of $4.94 \pm 0.42$ would have been predicted from the relationship shown in Pawellek \& Krivov (2015). The dust grains that constitute the disk would thus be larger than we expect from Pawellek \& Krivov (2015).

However, the minimum grain size inferred from our revised SED model is $3.25_{-1.00}^{+3.00} \mu \mathrm{m}$, consistent with the expectations of Pawellek \& Krivov (2015). We also infer a size distribution exponent of $q=3.55_{-0.15}^{+0.30}$. Our fit to the SED in the submillimeter is dominated by the far-infrared photometry, particularly the PACS $160 \mu \mathrm{m}$. In combination, the best-fit grain size and slope parameters result in a steeper millimeter SED than inferred from the $9 \mathrm{~mm}$ ATCA data point. A relatively bright point source resides at $10^{\prime \prime}$ from HD 105 in the ALMA image. While this could be responsible for contaminating the APEX/LABOCA $880 \mu \mathrm{m}$ data point, the ATCA beam was $5^{\prime \prime} \times 4^{\prime \prime}$, so it would be well separated from the disk at $9 \mathrm{~mm}$. Alternatively, stellar chromospheric emission could be contributing to the $9 \mathrm{~mm}$ flux measurement; enhanced emission (above that predicted from Rayleigh-Jeans extrapolation) has been observed at millimeter wavelengths for several stars (e.g., MacGregor et al. 2013; Liseau et al. 2015; Chavez-Dagostino et al. 2016). We might also infer that the dust grains have enhanced emissivity at submillimeter wavelengths, an inference supported by the relatively shallow millimeter spectral slope index $\beta=0.3$. Increasing the emissivity of dust grains at millimeter wavelengths can be achieved in many ways, including increasing the porosity or adopting a core-mantle model.

As an additional data point in the menagerie of resolved debris disks around Sun-like stars, we can examine the properties of the system in contrast to disks around stars of 
similar spectral type. The Jena catalog of resolved debris disks ${ }^{18}$ has 16 debris disk-host stars that fit this criterion, taken from various literature sources (Krist et al. 2010, 2012; Ertel et al. 2011; Eiroa et al. 2013; Marshall et al. 2014a; Soummer et al. 2014; Kennedy et al. 2015; Dodson-Robinson et al. 2016; Choquet et al. 2016; Lieman-Sifry et al. 2016; Moór et al. 2016; Morales et al. 2016). Among this cohort, the properties of HD 105's disk are fairly typical, having a radius of $\sim 90$ au (70-150 au), a typical dust temperature around $50 \mathrm{~K}(44-112 \mathrm{~K})$, and a fractional luminosity of a few $\times 10^{-4}\left(1 \times 10^{-5}-5 \times 10^{-3}\right)$. However, the constituents of this data set are heterogeneous, the disks having been resolved through various methods (optical, far-infrared, and millimeter) and the host stars having a wide variety of ages.

Through angular averaging, we have managed to obtain a detection of the disk in scattered light. The angular averaging method, used here for the first time, facilitated a detection of disk-scattered light and a deeper probe of the dust albedo than obtainable from standard analysis of the imaging observations through injection of a disk model; application of the angular averaging method to similar cases of continuum bright disks that remain undetected in scattered light is expected to yield further detections. The architecture of HD 105's disk bears a striking resemblance to that of both HD 207129 (Krist et al. 2010; Marshall et al. 2011; Löhne et al. 2012) and HD 377 (Choquet et al. 2016; Steele et al. 2016). The albedo determined from the scattered light brightness calculated here is comparable to that found for dust grains in imaged disks of comparable fractional luminosity around nominally similar stars, e.g., HD 207129 (albedo 0.05; Krist et al. 2010), and the properties of planetesimals in the solar system.

\section{Conclusions}

We have presented an analysis combining the interpretation of high-resolution stellar spectra alongside new and archival scattered light, far-infrared, and (sub-)millimeter imaging observations of HD 105 and its circumstellar disk. In combination, these observations have provided the basis for a more detailed interpretation of the system, including its architecture and evolutionary state.

From a Bayesian approach applied to stellar evolution models, we confirm its youth, with an age of $50 \pm 16 \mathrm{Myr}$. Additionally, we found activity indicators which are consistent with being at a pre-main-sequence evolutionary stage. The disk's planetesimal belt is spatially resolved at millimeter wavelengths. The best-fitting architecture is found to be a single annulus with a semimajor axis of 85 au and a width of $30 \mathrm{au}$ at a moderate inclination of $50^{\circ}$. The disk is more compact than would be predicted from application of simple stellar luminosity-disk radius relationships, suggesting that the dust grains comprising the disk are either large or efficient emitters at (sub-)millimeter wavelengths (e.g., through increased porosity; del Burgo et al. 2003).

There is no evidence of any offset between the planetesimal belt and the stellar position or any evidence of nonaxisymmetric structure in the disk, either of which might imply the presence of a low-mass companion interacting with the disk. No evidence of significant CO (2-1) line emission was found from the area of the disk, consistent with recent

\footnotetext{
18 http://www.astro.uni-jena.de/index.php/theory/catalog-of-resolveddebris-disks.html
}

discoveries of gas in young $(<60 \mathrm{Myr})$ debris disks being found in disks around A-type stars.

Using an angular averaging technique to measure the scattered light radial profile, we have obtained a first detection of HD 105's debris disk in scattered light, determining a value for the dust albedo between 0.15 and 0.06 . The application of this new technique to similar extant data sets, where the spatial extent of the disk is known from continuum emission but scattered light imaging has proven fruitless, should easily yield additional detections of debris disks in scattered light. This will provide an expanded pool of systems from which inferences can be drawn regarding their scattered light and thermal emission properties.

Comparison of the dust albedo inferred from the scattered light with simple grain models and simplifying assumptions with water ice/astronomical silicate compositions allows us to infer that the dust grains are consistent with either pure astronomical silicate such as is typically assumed for debris dust, or a moderately icy composition as might be expected for material lying well beyond the snow line of the system and consistent with SED modeling of a number of other debris disks.

We thank the anonymous referee for their constructive criticism that improved the manuscript.

Herschel is an ESA space observatory with science instruments provided by European-led Principal Investigator consortia and with important participation from NASA.

This paper makes use of the following ALMA data: ADS/ JAO.ALMA\#2012.1.00437.S. ALMA is a partnership of ESO (representing its member states), NSF (USA) and NINS (Japan), together with NRC (Canada) and NSC and ASIAA (Taiwan) and KASI (Republic of Korea), in cooperation with the Republic of Chile. The Joint ALMA Observatory is operated by ESO, AUI/NRAO, and NAOJ.

This research has made use of the SIMBAD database, operated at CDS, Strasbourg, France.

This research has made use of NASA's Astrophysics Data System.

This paper has made use of the Python packages ASTROPY (Astropy Collaboration et al. 2013, 2018), SCIPY (Jones et al. 2001), MATPLOTLIB (Hunter 2007), and HYPERION (Robitaille 2011).

J.P.M. acknowledges this research has been supported by the Ministry of Science and Technology of Taiwan under grants MOST107-2119-M-001-031-MY3 and MOST107-2119-M001-031-MY3.

E.C. acknowledges support from NASA through Hubble Fellowship grant HF2-51355 awarded by STScI, which is operated by AURA, Inc., for NASA under contract NAS526555, for research carried out at the Jet Propulsion Laboratory, California Institute of Technology. This work is based on data reprocessed as part of the ALICE program, which was supported by NASA through grants HST-AR-12652 (PI: R. Soummer), HST-GO-11136 (PI: D. Golimowski), HST-GO-13855 (PI: E. Choquet), and HST-GO-13331 (PI: L. Pueyo), and STScI Director's Discretionary Research funds. C.d.B. acknowledges that this work has been supported by Mexican CONACyT research grant CB-2012-183007.

G.M.K. is supported by the Royal Society as a Royal Society University Research Fellow. 
Pawellek, N., Krivov, A. V., Marshall, J. P., et al. 2014, ApJ, 792, 65

Perryman, M. A. C., Lindegren, L., Kovalevsky, J., et al. 1997, A\&A, 323, L49

Pollack, J. B., Hollenbach, D., Beckwith, S., et al. 1994, ApJ, 421, 615

Pueyo, L. 2016, ApJ, 824, 117

Ricci, L., Carpenter, J. M., Fu, B., et al. 2015, ApJ, 798, 124

Robitaille, T. P. 2011, A\&A, 536, A79

Rodigas, T. J., Debes, J. H., Hinz, P. M., et al. 2014, ApJ, 783, 21

Rodigas, T. J., Hinz, P. M., Leisenring, J., et al. 2012, ApJ, 752, 57

Schneider, G., Grady, C. A., Hines, D. C., et al. 2014, AJ, 148, 59

Schneider, G., Grady, C. A., Stark, C. C., et al. 2016, AJ, 152, 64

Schneider, G., Smith, B. A., Becklin, E. E., et al. 1999, ApJL, 513, L127

Schüppler, C., Krivov, A. V., Löhne, T., et al. 2016, MNRAS, 461, 2146

Schüppler, C., Löhne, T., Krivov, A. V., et al. 2014, A\&A, 567, A127

Soummer, R., Perrin, M. D., Pueyo, L., et al. 2014, ApJL, 786, L23

Soummer, R., Pueyo, L., \& Larkin, J. 2012, ApJL, 755, L28

Soummer, R., Sivaramakrishnan, A., Pueyo, L., Macintosh, B., \& Oppenheimer, B. R. 2011, ApJ, 729, 144
Spangler, C., Sargent, A. I., Silverstone, M. D., Becklin, E. E., \& Zuckerman, B. 2001, ApJ, 555, 932

Steele, A., Hughes, A. M., Carpenter, J., et al. 2016, ApJ, 816, 27

Tang, J., Bressan, A., Rosenfield, P., et al. 2014, MNRAS, 445, 4287

Thureau, N. D., Greaves, J. S., Matthews, B. C., et al. 2014, MNRAS, 445, 2558

Torres, C. A. O., da Silva, L., Quast, G. R., de la Reza, R., \& Jilinski, E. 2000, AJ, 120,1410

Tsantaki, M., Sousa, S. G., Santos, N. C., et al. 2014, A\&A, 570, A80

Valenti, J. A., \& Fischer, D. A. 2005, ApJS, 159, 141

van Leeuwen, F. 2007, A\&A, 474, 653

Wahhaj, Z., Milli, J., Kennedy, G., et al. 2016, A\&A, 596, L4

Weinberger, A. J., Becklin, E. E., Schneider, G., et al. 1999, ApJL, 525, L53

Wright, E. L., Eisenhardt, P. R. M., Mainzer, A. K., et al. 2010, AJ, 140, 1868

Wyatt, M. C., Kennedy, G., Sibthorpe, B., et al. 2012, MNRAS, 424, 1206

Zuckerman, B., \& Becklin, E. E. 1993, ApJ, 414, 793

Zuckerman, B., \& Webb, R. A. 2000, ApJ, 535, 959 